Wildy, P. \& HaRe, R. (1953). J. gen. Microbiol. 9, 216-225.

\title{
The Effect of Fatty Acids on the Growth, Metabolism and Morphology of the Anaerobic Cocci
}

\author{
BY P. WILDY AND R. HARE \\ Department of Bacteriology, St Thomas's Hospital Medical School, London
}

\begin{abstract}
SUMMARY: The sodium salts of a number of fatty acids had little, if any, effect on the metabolism or morphology of representative strains of anaerobic cocci of groups I, II, V and VI (Hare, Wildy, Billett \& Twort, 1952). However, strains of groups III and IV in the presence of an adequate concentration of fatty acid showed much greater activity than in media without substances of this nature; growth was very much better, gas was formed in larger amounts and organic acids (e.g. pyruvate) or carbohydrates (e.g. fructose) were attacked. The morphology of these group III and IV organisms was also affected in that the cocci were small $(0.5-0.8 \mu$.) and well formed in the presence of adequate concentrations of fatty acid, whereas in control preparations they were larger $(1 \cdot 2-1 \cdot 6 \mu$.) and sometimes had transverse septa.
\end{abstract}

In the course of investigations on the effect of various substances on the growth of anaerobic cocci, it was found that Tween $\mathbf{8 0}$ stimulated the growth of some strains of these organisms. Further experiments showed that a number of fatty acids also stimulated growth, and had marked effects on the morphology of the organisms and on their ability to ferment carbohydrates. These experiments form the subject of this communication.

\section{EXPERIMENTAL METHODS}

Strains studied. The collection and maintenance of the anaerobic cocci used was described by Hare et al. (1952). All the strains had been classified by their methods and representative strains were used for the tests.

Apparatus. Because of the danger that ordinary washing methods might fail to remove fatty acids from tubes used in previous experiments; all glassware was soaked in chromic + sulphuric acid for $24 \mathrm{hr}$. after cleaning and then washed with distilled water. There was also a danger that at oven temperatures fatty substances present in cotton-wool might contaminate the glassware (Wright, 1934). For this reason previously dry-sterilized tubes were not used; instead, clean but unsterile tubes were filled with medium, plugged and sterilized by autoclaving or steaming.

Media. The basal medium employed consisted of: $2 \%(w / v)$ peptone (Evans); 0.2\% (w/v) yeast extract (Difco); $0.1 \%(w / v)$ sodium mercaptoacetate (thioglycollate). After solution in distilled water and the addition of fatty acids, sugars or organic acids as required, sufficient $\mathrm{N}-\mathrm{NaOH}$ was added to bring the medium to the required $\mathrm{pH}$ value. The solution was then brought to boil, immediately filtered through hard filter paper and tubed in $4 \mathrm{ml}$. quantities in $5 \times \frac{1}{2}$ in. tubes. When the medium contained carbohydrate, each tube was capped with hot vaseline and the batch sterilized in the steamer on 
three successive days. When salts of organic acids only were added, the tubes were autoclaved at $15 \mathrm{lb}$./sq. in. for $30 \mathrm{~min}$. and immediately capped with hot vaseline.

When the effect of fatty acids was under investigation the above medium was made with the volume of distilled water decreased by one-tenth, and made up to one volume by the addition of fatty acid solution (or distilled water for controls). The fatty acids were made up as $0.01 \mathrm{M}$-solution in $0.01 \mathrm{~N}$-sodium hydroxide and held at $100^{\circ}$ in a boiling water bath until homogeneous. While still hot, tenfold dilutions were made in distilled water and one volume of these dilutions was added to 9 volumes of medium as described above.

Inoculation of tubes, measurements of opacity, gas formation and final $\mathrm{pH}$ value. The methods employed were those described by Hare et al. (1952), the cultures being incubated for 7 days, the opacity determined on the scale of Brown \& Kirwan (1914-15), the length of gas column recorded in millimetres and the final $\mathrm{pH}$ value determined colorimetrically on porcelain tiles.

\section{RESULTS}

An initial experiment with the addition of Tween 80 showed that while the strains of groups I, II, V and VI (Hare et al. 1952) were unaffected by concentrations of this substance ranging from $0.001 \%(w / v)$ to $10 \%(w / v)$, those of groups III and IV grew much more profusely in the higher concentrations. A similar experiment was then carried out with representative strains of all the groups in broth containing varying concentrations of sodium oleate and $1 \%$ pyruvate to stimulate growth (Hare et al. 1952). The results are given in Table 1. There was no detectable effect on the strains of groups V and VI, and the only action on group I strains was slight inhibition of gas formation in the highest concentration of oleate. There was also some inhibition of growth and a slight diminution in the quantity of gas produced by group II strains. But the most marked effect was on the strains of groups III and IV. In the case of group III, one strain grew very much better and produced considerably greater quantities of gas in the presence of oleate, particularly at $100 \mu \mathrm{m}$. The second strain also produced larger quantities of gas, and although the opacity reading on the 8th day was of the same order as that of the control tube, this was to some extent misleading because opacities of 5 or 6 were actually obtained on the 4 th day with this strain. It must be presumed that the lower reading on the 8th day was due to autolysis of the organisms with continued incubation. This phenomenon has repeatedly been observed with this strain. The effect of sodium oleate on strains of group IV was more dramatic, particularly at $100 \mu \mathrm{M}$, which caused marked stimulation of the growth of all strains and the production of gas by some of these strains, a character not previously observed with strains of this group.

A similar experiment was then carried out, representative strains from each of the groups being inoculated into media containing $1 \%$ pyruvate and different concentrations of the sodium salts of purified capric, lauric, myristic, palmitic, stearic or oleic acids. The results in so far as they concerned groups I, II, $\mathrm{V}$ and VI showed that none of the fatty acids at concentrations from $1 \mu \mathrm{M}$ to 
Table 1. The behaviour of representative strains of 6 groups of anaerobic cocci in different concentrations of sodium oleate in $1 \%(w / v)$ pyruvate broth

\begin{tabular}{|c|c|c|c|c|c|c|}
\hline \multirow[b]{2}{*}{ Group } & \multirow{2}{*}{$\begin{array}{l}\text { No. of } \\
\text { strains } \\
\text { tested }\end{array}$} & \multicolumn{5}{|c|}{ Oleate concentration } \\
\hline & & $\mathbf{0}$ & $1 \mu \mathrm{M}$ & $10 \mu \mathrm{M}$ & $100 \mu \mathrm{M}$ & $1000 \mu \mathrm{M}$ \\
\hline I & 2 & $\begin{array}{l}\text { Gas: } 2,17-20 \\
\text { Opacity: } 2,3\end{array}$ & $\begin{array}{c}2,17-20 \\
2,3\end{array}$ & $\begin{array}{c}2,18-20 \\
2,3\end{array}$ & $\begin{array}{c}2,21-22 \\
2,3\end{array}$ & $\begin{array}{c}2,0-17 \\
2,3\end{array}$ \\
\hline II & 2 & $\begin{array}{l}\text { Gas: } \quad 2,55-80 \\
\text { Opacity: } 2,4\end{array}$ & $\begin{array}{c}2,60-72 \\
2,4\end{array}$ & $\begin{array}{l}2,72-85 \\
1,2 \\
1,4\end{array}$ & $\begin{array}{l}2,50-70 \\
1,2 \\
1,4\end{array}$ & $\begin{array}{c}2,35-39 \\
1,3 \\
1,4\end{array}$ \\
\hline III & 2 & $\begin{array}{l}\text { Gas: } \quad 2,35-38 \\
\text { Opacity: } 1,<1 \\
1,1\end{array}$ & $\begin{array}{c}2,30-45 \\
1,<1 \\
1,2\end{array}$ & $\begin{array}{l}2,52-87 \\
1,1 \\
1,5\end{array}$ & $\begin{array}{c}2,45-77 \\
1,<1 \\
1,6\end{array}$ & $\begin{array}{c}2,31-54 \\
1,<1 \\
1,6\end{array}$ \\
\hline IV & 8 & $\begin{array}{ll}\text { Gas: } & 8,0 \\
\text { Opacity: } & 8,<1\end{array}$ & $\begin{array}{c}8,0 \\
7,<1 \\
1,1\end{array}$ & $\begin{array}{l}8,0 \\
6,1 \\
2,2\end{array}$ & $\begin{array}{c}5,0 \\
3,<1-18-22 \\
1,3 \\
4,4 \\
2,5 \\
1,6\end{array}$ & $\begin{array}{c}6,0 \\
2,11-20 \\
1,3 \\
4,4 \\
1,5 \\
2,6\end{array}$ \\
\hline $\mathbf{V}$ & 2 & $\begin{array}{l}\text { Gas: } 2,68-71 \\
\text { Opacity: } 2,6\end{array}$ & $\begin{array}{c}2,71-72 \\
2,6\end{array}$ & $\begin{array}{l}2,70-74 \\
2,6\end{array}$ & $\begin{array}{l}2,70-77 \\
2,6\end{array}$ & $\begin{array}{c}2,0-78 \\
2,6\end{array}$ \\
\hline VI & 2 & $\begin{array}{ll}\text { Gas: } & 2,0 \\
\text { Opacity: } & 1,<1 \\
& 1,1\end{array}$ & $\begin{array}{c}2,0 \\
2,<1\end{array}$ & $\begin{array}{c}2,0 \\
2,<1\end{array}$ & $\begin{array}{c}2,0 \\
1,<1 \\
1,1\end{array}$ & $\begin{array}{c}2,0 \\
2,<1\end{array}$ \\
\hline
\end{tabular}

In this table and the succeeding one, the figures relating to 'gas' denote the length (in mm.) of the column of gas collecting between the vaseline seal and the surface of the medium. The figure before the comma denotes the number of strains and the numbers after the readings obtained in this particular experiment.

Those relating to opacity denote the tube on Brown \& Kirwan's scale whose opacity was the nearest to that obtained. The figure before the comma denotes the number of strains and that after the comma, the opacity reached in this experiment.

$1000 \mu \mathrm{M}$ had any marked effect on the growth of the strains; but there were marked effects on strains of groups III and IV (see Table 2). Both strains of group III were stimulated by all the compounds except caprate and palmitate. Caprate had no detectable action on strains of group IV, whereas laurate, stearate and oleate were extremely active, with palmitate and myristate occupying an intermediate position. In other experiments, the same strains, together with representative strains of the other groups, were tested under similar conditions in the presence of sodium salts of formic, acetic, propionic, butyric, valeric, caproic, heptoic, caprylic, pelargnonic, margaric, nonadecylic or behenic acids (purity and freedom from contamination by other fatty acids not guaranteed). With compounds containing fewer than 9 carbon atoms per molecule, no effects were obtained, but some stimulation of strains of groups III and IV occurred in the presence of pelargonate, nonadecylate or behenate.

Thus it is clear that a suitable concentration of certain fatty acids is needed if good growth of strains of groups III and IV is to be obtained. Although compounds having this effect possess 12 to 18 carbon atoms per molecule, there was no evidence that their activity is linked with the length of their chains; laurate with 12 carbon atoms and stearate with 18 carbon atoms were 


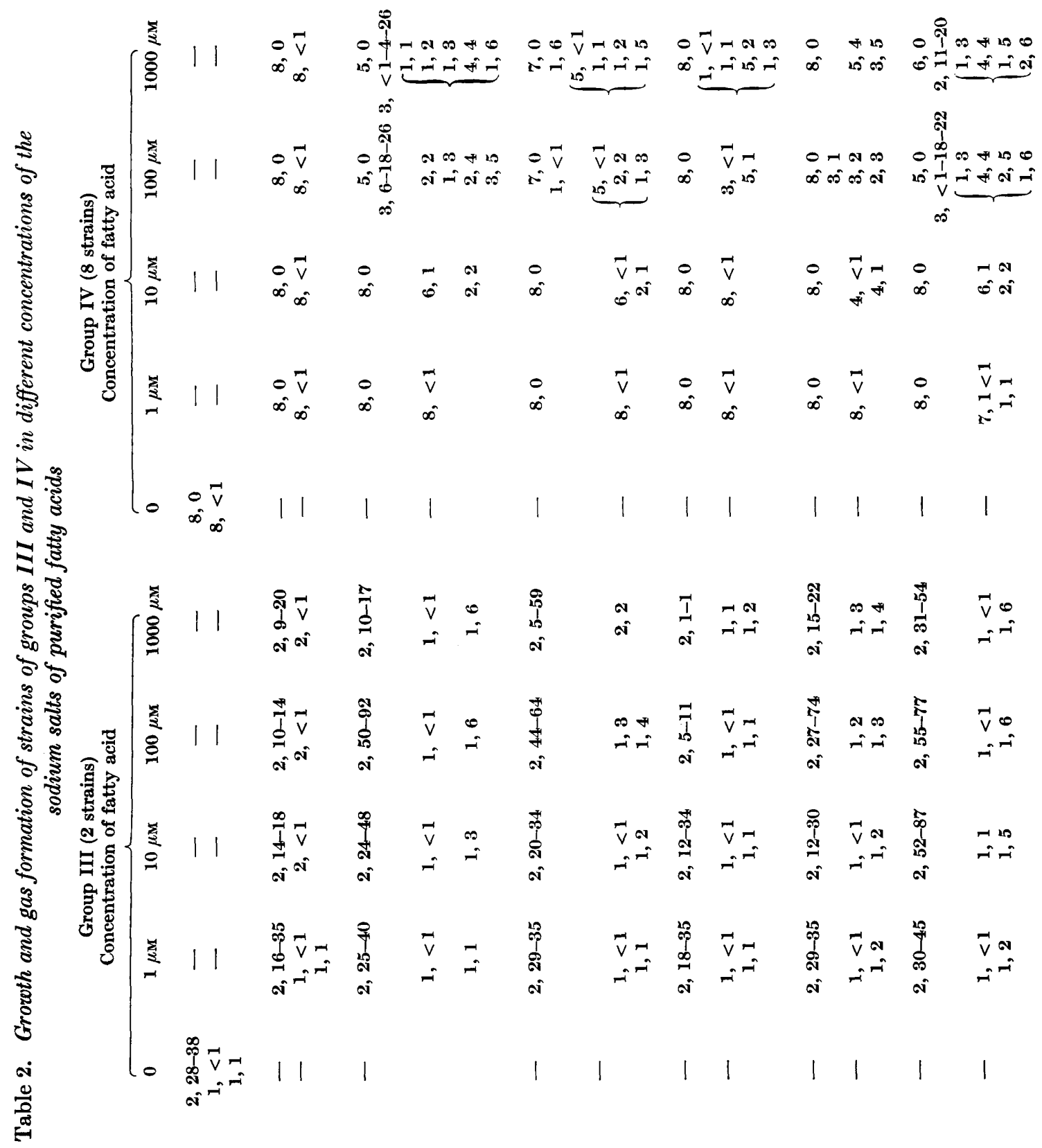

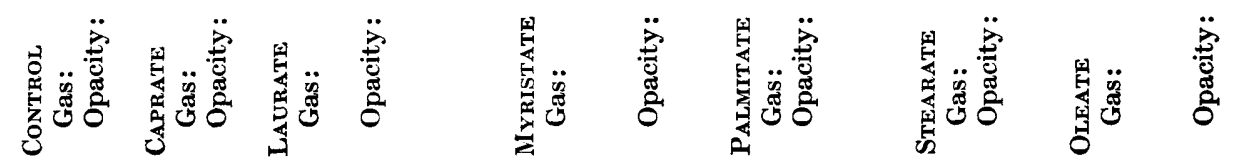


very active, intermediate compounds in the series, such as myristate with 14 carbon atoms and palmitate with 16 carbon atoms were much less so. This comparison between the activity of these acids has been repeated several times with identical results. It seems improbable that the effect was due to impurities in the fatty acids used; the effects recorded in Table 2 were obtained with acids which were 99-100\% pure, but if any of the effects observed with a particular compound were due to contamination by any of the others, the contaminating acid would need to have been present in a concentration of at least $10 \%$ of the material. Nevertheless, it is conceivable that some quite different growth factor of high potency might have to be present in some of the acids.

\section{The effect of fatty acids on the metabolism of organic acids}

Hare et al. (1952) showed that strains of groups I, II, III and V were able to utilize certain organic acids, better growth and larger quantities of gas being produced in their presence than in control media not containing them. Experiments were therefore carried out to investigate whether sodium oleate at $355 \mu \mathrm{M}$ had any effect on these reactions. There was no detectable effect on the behaviour of strains of groups I, II, V and VI towards these substances, but oleate stimulated the growth of both groups III and IV in pyruvate and more gas was produced by group III strains.

In other experiments, using $1 \%(\mathrm{w} / \mathrm{v})$ pyruvate and different concentrations of oleate it was found that in the presence of $355 \mu \mathrm{M}$ or $35.5 \mu \mathrm{M}$-oleate, the $\mathrm{pH}$ of the medium fell from the initial $7 \cdot 0$ to $6 \cdot 2-6 \cdot 4$ with group III, and to 5.6-6.0 in the case of group IV. With $3.5 \mu \mathrm{M}$-oleate or none, there was little, if any, fall in the pH value. Further investigations, using Simon \& Piaux's (1924) qualitative method, showed that with both groups all the pyruvate had been utilized in the presence of the higher concentrations of fatty acids but little, if any, in the lower concentrations or in control tubes.

\section{Effect of fatty acids on fermentation of carbohydrates}

Hare et al. (1952) showed that strains of groups I and VI fermented a number of carbohydrates, whereas those of other groups had little or no activity. This inability to ferment may have been due in part to the fact that growth was very poor. Experiments were therefore carried out to determine whether the better growth obtainable in the presence of a fatty acid (e.g. oleate) might induce fermentation. The medium employed consisted of $2.0 \%(w / v)$ peptone; $0 \cdot 2 \%(w / v)$ yeast extract; $0 \cdot 1 \%(w / v)$ sodium mercaptoacetate (thioglycollate); and $1 \%$ glucose, maltose, fructose, sucrose or galactose. A control without carbohydrate was also included. Parallel series were set up, one without and the other with $355 \mu \mathrm{M}$-oleate. The tubes were incubated for 7 days and the volume of gas produced, the opacity of the culture and the $\mathrm{pH}$ value determined.

The general behaviour of groups I, II, V and VI was very little affected by the presence of oleate. Marked differences were observed with groups III and IV. With group III Hare et al. (1952) showed that in the medium they 
used (containing $5 \%$ peptone and $1 \%$ carbohydrate) these strains depressed the $\mathrm{pH}$ value slightly. A similar small decrease in $\mathrm{pH}$ value in media without oleate was obtained in these experiments (from $\mathrm{pH} 8.0$ to 6.8-7.0) but in presence of oleate the $\mathrm{pH}$ values fell from an initial 8.0 to 5.4-5.6 in glucose, maltose, fructose or sucrose; growth was then very much better and large quantities of gas were produced. Group IV strains in the presence of oleate were similarly able to ferment fructose, the final $\mathrm{pH}$ value being 5・8-6.4 with 7 strains, but no gas was produced. In control media without oleate, these strains grew very badly and with no sign of fermentation.

In a second experiment using $1 \%$ fructose and different concentrations of oleate, it was found that with strains of group III as little as $3.55 \mu$ M-oleate sufficed to produce detectable fermentation whereas with strains of group IV 35.5 $\mu$ M-oleate was necessary.

\section{Growth on agar}

Strains of groups III and IV grew very sparsely in broth without added fatty acid. But it was repeatedly observed that when the same broth was used to prepare agar plates, excellent growths were obtained after incubation for $48 \mathrm{hr}$. in a McIntosh \& Fildes jar. Because relatively small quantities of fatty acids can stimulate growth, it seemed possible that substances of this nature were present as impurities in agar and were responsible for the better and more luxuriant growth on solid medium. Medium containing $1 \%(\mathrm{w} / \mathrm{v})$ peptone, $0.2 \%(w / v)$ Difco yeast extract and $2.0 \%(w / v)$ Difco agar powder was made up and Norit (British Drug Houses Ltd.) added at different concentrations. The medium was sterilized by autoclaving. Representative strains, as $48 \mathrm{hr}$. broth cultures, were streaked over the plates which were incubated for $48 \mathrm{hr}$. in a McIntosh \& Fildes jar. Strains of groups I, II and VI were indifferent, those of group $\mathrm{V}$ grew better on the agar containing charcoal and those of group III and IV were almost completely inhibited when Norit was present in a concentration as low as $0.012 \%(w / v)$.

To test whether toxic material had been extracted from the Norit, agar plates were prepared containing $\mathbf{0 \cdot 2} \%(\mathrm{w} / \mathrm{v})$ Norit. A ditch was then cut to one side of the plate and filled with agar containing the same concentration of Norit and $355 \mu \mathrm{M}$-oleate as well. As in the preceding experiment growth failed over most of the plates, but good growth was obtained over the ditch and in a zone of 8-100 mm. adjacent to it. It is, therefore, more probable that the failure of groups III and IV organisms to grow on agar containing Norit was due to the removal of fatty acids from the agar by the Norit and not to the presence of toxic material derived from the Norit.

Microscopic appearances of cocci in cultures with and without oleate

Microscopical examination of the sediment in tubes containing broth with different amounts of fatty acid showed that the presence or absence of the fatty acid made no difference to the arrangement or sizes of the cocci or groups I, II, V and VI; but with organisms of groups III and IV there was a definite correlation between size of cocci and fatty acid concentration. An experiment 
was then set up in which different concentrations of oleate were added to broth medium with and without $1 \%$ fructose. They were inoculated with representative strains of groups III and IV and incubated. Films were made of the deposit in the tubes after incubation for 24,48 and $144 \mathrm{hr}$. The films were fixed by heat, cleared with acid ethanol and stained with carbol-fuchsin $(0.1 \%)$ and examined; individual cocci were measured with an eyepiece micrometer.

The appearances after 24, 48 and $144 \mathrm{hr}$. incubation were similar; in the account given below, those seen after $48 \mathrm{hr}$. are described. Group III strains multiplied in the form of diplococci with the contiguous sides slightly flattened, but the size of the individual cocci varied with the concentration of oleate. In $355 \mu$ M-oleate they varied from 0.6 to $0.8 \mu$. diam. with a few of $1.0 \mu$.; in $35.5 \mu \mathrm{M}$ and $3.55 \mu \mathrm{M}$-oleate, they were 1.0 to $1.2 \mu$. diam.; without oleate the cocci were as large as 1.2 to $1.6 \mu$. diam. The presence of fructose made no detectable difference to the sizes or arrangement of the organisms despite the fact that the growth was heavier.

On the whole, a similar picture was observed with group IV strains, the principal difference being that in absence of oleate or with only $0.355 \mu \mathrm{M}$ or 3.55 $\mu$ M-oleate, there were many poorly stained ghosts, although some of them had quadrants or crescents which were well stained. There was about the same number of well-stained large cocci (1.4 to $1.6 \mu$. diam.) and very few cocci 1.0 $\mu$. diam. With 35.5 $\mu \mathrm{M}$-oleate there was a range of sizes, some cocci being 1.0 to $1.2 \mu$. diam. but many were 0.5 to $0.8 \mu$. In $355 \mu \mathrm{M}$-oleate small cocci from 0.5 to $0.6 \mu$. diam. were everywhere in evidence; the larger forms were not easy to find, but some of $1 \cdot 0 \mu$. diam. were present. There was much less tendency for the cocci to appear as diplococci than with group III organisms but, as with that group, fructose made no difference to the appearance of the individual organisms despite the fact that much better growth was obtained in its presence (see Pl. 1, figs. 1, 2, 3, 4, 5, 6). Thus, the diameter of group III and group IV cocci varied from $0.5 \mu$. to $1.6 \mu$. according to the fatty acid content of the medium.

The general appearance of the large cocci in films from cultures in which there was no oleate or only traces of it suggests that they were starvation cells, able to enlarge but incapable of carrying through the process of division to completion; preparations in which the cell wall was stained with methyl violet after mordanting with tannic acid certainly showed the presence of transverse septa in some cells (see Pl. 1, figs, 7, 8, 9). There was no evidence that all the large forms were multicellular. These large forms are comparable to those seen when some species of bacteria are grown in Mg-deficient media (Webb, 1948), in sub-lethal concentrations of dyes, such as methyl-violet (Ainley-Walker \& Murray, 1904), or in penicillin (Gardner, 1940).

\section{DISCUSSION}

From these experiments it seems obvious that in the presence of certain fatty acids, e.g. oleate, the amount of growth, ability to attack carbohydrates or organic acids, formation of gas and morphology of the cocci of groups III and 
IV, may be considerably modified. Although fatty acids have been known for some time to inhibit the growth of some species of organism, they have also been recognized as growth factors for other species; Fleming (1909), for instance, observed this in the acne bacillus, Dubos (1947) with Mycobacterium tuberculosis and Thoma \& Peterson (1950) with certain clostridia. Williams \& Fieger (1946) were unable to detect any connexion between the structure of a fatty acid and its activity as a growth-enhancing agent for Lactobacillus casei and considered that its activity was due principally to its physical properties. On the other hand, Boughton \& Pollock $(1952,1953)$ observed that the length of chain and the position of the double bond in the fatty acid molecule were of importance in the promotion of the growth of a diphtheroid organism; they obtained data which suggested that the fatty acid formed a constituent part of the cell. Furthermore, unsaturated acids were more effective than saturated acids. Boughton \& Pollock suggested that the growth-enhancing activities of a saturated fatty acid was due to its ability to overcome the toxicity of excessive amounts of unsaturated fatty acid present in the medium.

The data in this paper are insufficient to show the way in which fatty acids increase so very considerably the growth and metabolic activities of certain of the anaerobic cocci. Although the most effective acids are those which are surface active, it seems improbable that their function is to break up masses of cells, for there is no greater degree of bunching or agglomeration of the cells when oleate is absent or deficient than when it is present in sufficient concentration. On the other hand, in media deficient in fatty acid, individual cells are very large indeed and although many of them possess septa, the two components are each larger than one cell which has grown in an adequate concentration of oleate. This appearance suggests that in such cells there has been elaboration of sufficient foodstuffs and the necessary enzymes for the first phases of division, but that the later stages cannot be carried through. Since somewhat similar appearances are seen in cells subjected to sub-lethal concentrations of antibiotics or dyes, it seems probable that lack of an essential metabolite is responsible. On the evidence at present available it is impossible to say whether this metabolite is the fatty acid itself or whether the latter can so alter the permeability of the cell as to permit better absorption.

If fatty acids are of importance in the metabolism of the anaerobic cocci, it follows that the concentration of substances of this nature in the media used for their growth becomes a matter of considerable importance. There seems little doubt that the basal liquid medium we used was deficient in such substances; when incorporated in agar the growth-supporting qualities of the medium became so much better as to suggest that this was due to fatty acids present in the agar as impurities. Ley \& Mueller (1946) showed that material having the chemical characteristics of a fatty acid can be extracted from agar and Pollock (1947, 1948) has also, to some extent, confirmed the presence of such substances in agar. Pollock found that the addition of charcoal to agar inhibited the growth of a corynebacterium which required a fatty acid, while the growth of a strain of Haemophilus pertussis which was inhibited by the 
presence of fatty acids, only grew on agar to which charcoal had been added. In our experiments, the presence of charcoal prevented growth of strains of groups III and IV on agar medium, but the same medium supported growth when oleate was allowed to diffuse into it. Although this suggests that oleate is a growth requirement for these strains, it does not preclude the possibility that the oleate had displaced some other metabolite from the surfaces of the carbon particles. Attempts to remove the charcoal in order to settle this point were only partially successful, but there were no indications that if its complete removal had been achieved, the results would have been different.

It is possible that very small quantities of fatty acid may be present in the peptone used, since Hare et al. (1952) obtained better growth of strains of groups III and IV in the presence of $5 \%(w / v)$ peptone than in $2 \%(w / v)$, and found that, although the diameter of the strains of group IV varied from 0.4 to $0.8 \mu$, there were no large forms of the kind regularly produced in $2 \%$ (w/v) peptone without added fatty acid. The large forms were observed, however, with group III strains in the $5 \%(\mathrm{w} / \mathrm{v})$ peptone media. In view of the evident importance of fatty acid concentration in modifying the growth, fermentation reactions and morphology of anaerobic cocci belonging to groups III and IV, it is obvious that unless the media used are carefully standardized, these criteria are worthless for purposes of classification.

Our thanks are due to Messrs Lever Bros. for the gift of the samples of purified fatty acids, to Mr Albert Clark for the photographs, and to Mrs D. N. Twort and Mr R. Priddy for technical assistance. One of us (P. W.) was in receipt of a grant from the Leonard S. Dudgeon and Michael and Sidney Herbert Funds, St Thomas's Hospital Medical School.

\section{REFERENCES}

Ainley-Walker, E. W. \& Murray, W. (1904). The effect of certain dyes upon the cultural characters of the Bacillus typhosus and some other micro-organisms. Brit. med. J. 2, 16.

Boughton, B. W. \& Pollock, M. R. (1952). Long chain unsaturated fatty acids as essential bacterial growth factors. Biochem. J. 50, xxxi.

Boughton, B. W. \& Pollock, M. R. (1953). Long chain unsaturated fatty acids as essential bacterial growth factors: further studies with Corynebacterium $\mathbf{Q}$. Biochem. J. 53, 261.

Brown, K. C. \& Kirwan, E. W. O. G. (1914-15). Standardization of bacterial suspensions by opacity. Indian J. med. Res. $2,763$.

DuBos, R. J. (1947). The effect of lipoids and serum albumen on bacterial growth. J. exp. Med. 85, 8.

Fleming, A. (1909). On the aetiology of acne vulgaris and its treatment by vaccines. Lancet, $\mathrm{i}, 1035$.

Gardner, A. D. (1940). Morphological effects of penicillin on bacteria. Nature, Lond. 146, 837.

Hare, R., Windy, P., Billete, F. S. \& Twort, D. N. (1952). The anaerobic cocci: gas formation, fermentation reactions, sensitivity to antibiotics and sulphonamides. Classification. J. Hyg., Camb. 50, 295.

KuIENeberger, E. (1934). The colonial development of the organisms of pleuropneumonia and agalactia on serum agar and variations of the morphology under different conditions of growth. J. Path. Bact. 39, 409. 
Journal of General Microbiology, Vol. 9, No. 2

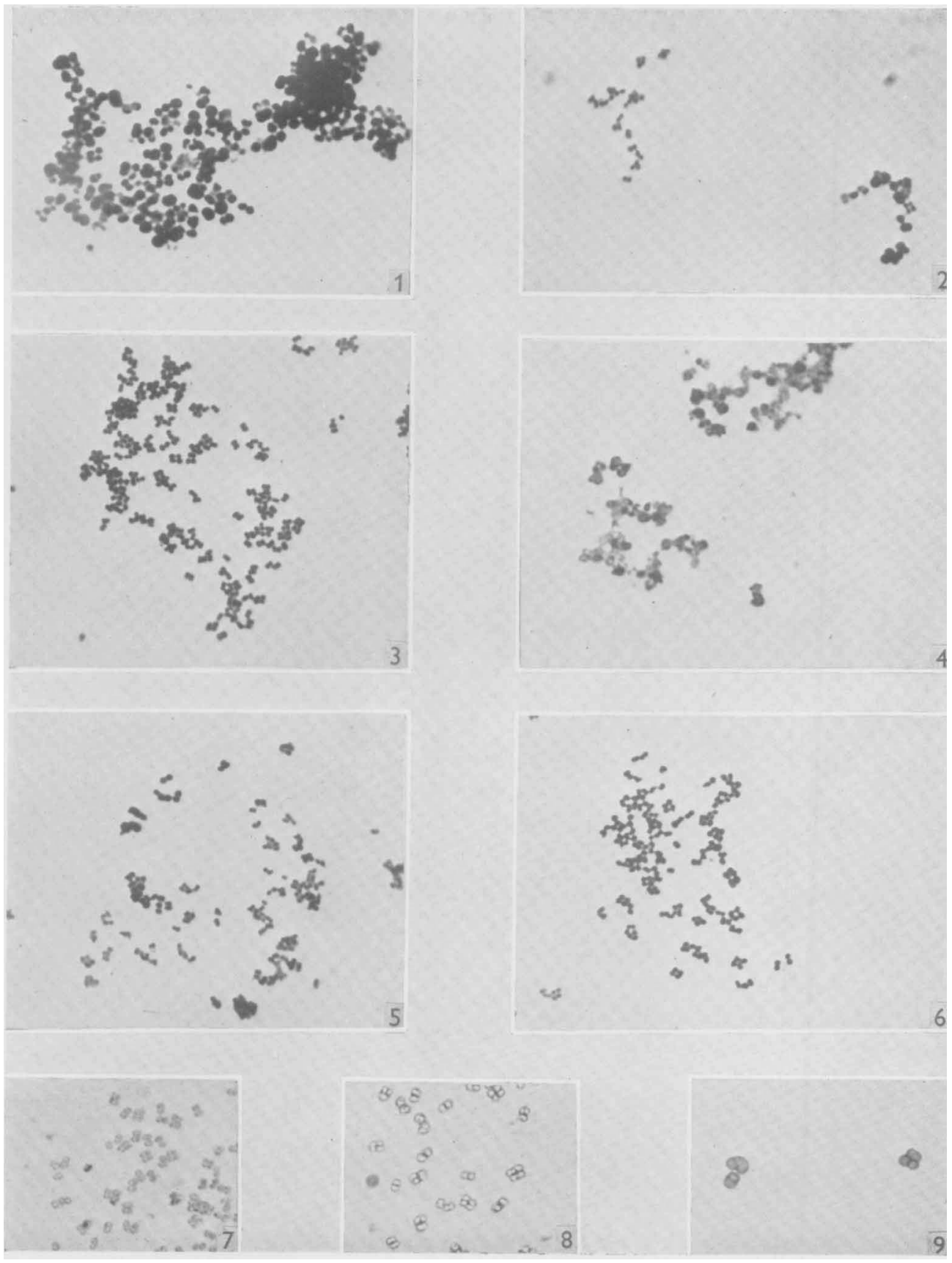

P. Wildy \& R. Hare-Effect of fatty acids on anaerobic cocci. Plate 1 
LEY, H. L. \& Mueller, J. H. (1946). On the isolation from agar of an inhibitor for Neisseria gonorrhoeae. J. Bact. 52, 453.

Pollock, M. R. (1947). The growth of $H$. pertussis on media without blood. Brit. J. exp. Path. 28, 295.

PoLLock, M. R. (1948). A case of bacterial symbiosis based on the combined growthstimulating and growth-inhibitory properties of long-chain unsaturated fatty acids. J. gen. Microbiol. 2, xxiii.

Roвinow, C. (1943-44). Cytological observations on Bact. coli, Proteus vulgaris and various aerobic spore forming bacteria with special reference to the nuclear structures. J. Hyg., Camb. 43, 413.

Simon, L. J. \& Praux, L. (1924). Sur la caractérisation et le dosage de petits quantités d'acide pyruvique. Bull. Soc. Chim. biol. Paris, 6, 477.

Thoma, R. W. \& Peterson, W. H. (1950). Biotin and arginine replacements in the nutrition of Clostridium sporogenes. J. Bact. 60, 39.

WEBB, M. (1948). The influence of magnesium on cell division. 1. The growth of Clostridium welchii in complex media deficient in magnesium. J.gen. Microbiol. 2,275 .

Wilhiams, V. R. \& Fieger, E. A. (1946). Oleic acid as a growth stimulant for Lactobacillus casei. J. biol. Chem. 166, 335.

WrIGHT, H. D. (1934). A substance in cotton wool inhibitory to the growth of the pneumococcus. J. Path. Bact. 38, 499.

\section{EXPLANATION OF PLATE}

$$
\text { (Magnification } \times \text { 1080) }
$$

Figs. 1-6. Group IV strain. Sediment from $2 \%$ peptone broth cultures, fixed by heat, cleared with acid ethanol and stained with carbolfuchsin $0 \cdot 1 \%$.

Fig. 1. $48 \mathrm{hr}$. incubation. No oleate.

Fig. 2. $48 \mathrm{hr}$. incubation. $35 \mu \mathrm{m}$-oleate.

Fig. 3. $48 \mathrm{hr}$. incubation. $355 \mu \mathrm{M}$-oleate.

Fig. 4. $144 \mathrm{hr}$. incubation. No oleate.

Fig. 5. 144 hr. incubation. $35 \mu \mathrm{M}$-oleate.

Fig. 6. $144 \mathrm{hr}$. incubation. $355 \mu \mathrm{m}$-oleate.

Figs. 7-9. Group IV strain. Impression preparations from agar plates containing $0 \cdot 2 \%(w / v)$ Norit. Ditch cut to one side and filled with agar containing $0.2 \%(w / v)$ Norit and $355 \mu \mathrm{m}$-oleate. Inoculated by streak across and incubated anaerobically for $48 \mathrm{hr}$. Fixed with Bouin's fluid (Klieneberger, 1934), mordanted for $2 \mathrm{hr}$. with $10 \%$ tannic acid and stained with $0.2 \%$ crystal violet (Robinow, 1943-44). Mounted in balsam.

Fig. 7 . Cocci over the ditch.

Fig. 8. Cocci from area $5 \mathrm{~mm}$. from ditch.

Fig. 9. Cocci from area $10-15 \mathrm{~mm}$. from ditch. 